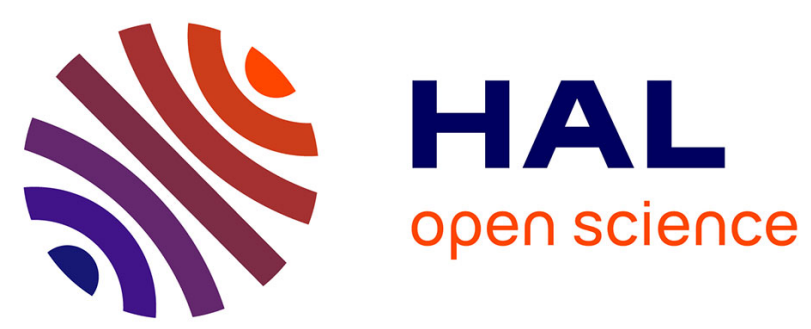

\title{
Extracorporeal gas exchange for acute respiratory failure in adult patients: a systematic review
}

\author{
Matthieu Schmidt, Carol Hodgson, Alain Combes
}

\section{To cite this version:}

Matthieu Schmidt, Carol Hodgson, Alain Combes. Extracorporeal gas exchange for acute respiratory failure in adult patients: a systematic review. Critical Care, 2015, 19, pp.99. 10.1186/s13054-0150806-z . hal-01261695

\section{HAL Id: hal-01261695 \\ https://hal.sorbonne-universite.fr/hal-01261695}

Submitted on 25 Jan 2016

HAL is a multi-disciplinary open access archive for the deposit and dissemination of scientific research documents, whether they are published or not. The documents may come from teaching and research institutions in France or abroad, or from public or private research centers.
L'archive ouverte pluridisciplinaire HAL, est destinée au dépôt et à la diffusion de documents scientifiques de niveau recherche, publiés ou non, émanant des établissements d'enseignement et de recherche français ou étrangers, des laboratoires publics ou privés. 


\title{
Extracorporeal gas exchange for acute respiratory failure in adult patients: a systematic review
}

\author{
Matthieu Schmidt ${ }^{1}$, Carol Hodgson ${ }^{2}$ and Alain Combes $^{1 *}$
}

\begin{abstract}
This article is one of ten reviews selected from the Annual Update in Intensive Care and Emergency Medicine 2015 and co-published as a series in Critical Care. Other articles in the series can be found online at http://ccforum.com/series/annualupdate2015. Further information about the Annual Update in Intensive Care and Emergency Medicine is available from http://www. springer.com/series/8901.
\end{abstract}

\section{Introduction}

Mechanical ventilation remains the cornerstone of respiratory support for patients with acute respiratory failure. However, high pressure and volume associated with tidal ventilation are known to aggravate lung injury in this setting [1]. Furthermore, profound gas-exchange abnormalities threatening patients' lives can occur in the most severe forms of the disease despite recourse to conventional salvage therapies [2,3]. Extracorporeal gas exchange devices, i.e., venovenous extracorporeal membrane oxygenation (ECMO) and extracorporeal carbon dioxide removal $\left(\mathrm{ECCO}_{2} \mathrm{R}\right)$, were developed more than 40 years ago $[4,5]$ to rescue these dying patients. Whereas venovenous ECMO provides complete extracorporeal blood oxygenation and decarboxylation using high blood flows (4-6 1/min) and large (20-30 Fr) cannulas [6-9], efficient extracorporeal $\mathrm{CO}_{2}$ removal (with minimal blood oxygenation) can be achieved with $\mathrm{ECCO}_{2} \mathrm{R}$ devices using limited extracorporeal blood flow (0.4-1 l/min) and thin double lumen venous catheters (14-18 Fr) [10,11], because $\mathrm{CO}_{2}$ clearance is more effective than oxygenation due to the greater solubility and more rapid diffusion of $\mathrm{CO}_{2}$ [12]. Extracorporeal gas exchange devices also permit 'ultraprotective' mechanical ventilation with further

\footnotetext{
* Correspondence: alain.combes@psl.aphp.fr

'Université Pierre et Marie Curie, Medical-Surgical Intensive Care Unit, iCAN, Institute of Cardiometabolism and Nutrition, Hôpital de la Pitié-Salpêtrière, Assistance Publique-Hôpitaux de Paris, Paris, France

Full list of author information is available at the end of the article
}

reduction of volume and pressure, which may ultimately enhance lung protection and improve clinical outcomes for patients with acute respiratory distress syndrome (ARDS). However, results of trials evaluating extracorporeal gas exchange for respiratory failure performed in the 1970s, 80s and 90 s were often disappointing $[13,14]$. In recent years, major technological advances have occurred and the latest generation extracorporeal gas exchange devices, with polymethylpentene hollow-fiber membrane lungs and Mendler-designed centrifugal pumps offer lower resistance to blood flow, have smaller priming volumes, higher effective gas exchange properties and are coated with more biocompatible materials.

The successful use of ECMO for the most severe ARDS cases associated with the recent influenza A (H1N1) pandemic, in whom conventional ventilation was not successful [15-17], and positive results of the randomized Conventional ventilatory support versus Extracorporeal membrane oxygenation for Severe Adult Respiratory failure (CESAR) trial [18] have been associated with a steep increase in the number of VV-ECMO procedures performed in very recent years (Figure 1). In addition, a proof-of-concept study suggested that the very low tidal volume ventilation (3.5-5 ml/kg of predicted body weight) permitted by $\mathrm{ECCO}_{2} \mathrm{R}$ can improve pulmonary protection and decrease pulmonary inflammation in ARDS patients [19] and a recent randomized trial suggested that this strategy may be associated with better outcomes for moderate to severe ARDS patients [11].

The aim of this systematic review was to analyze studies reporting indications, associated complications and shortand long-term outcomes of extracorporeal gas exchange in adult patients with acute respiratory failure. It may ultimately help critical care physicians and researchers select better candidates for extracorporeal gas exchange and to design future observational and randomized clinical trials to evaluate these techniques. 


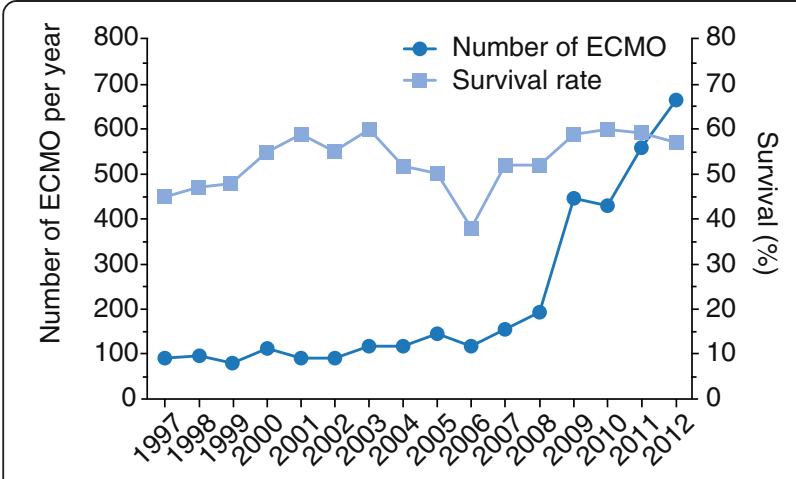

Figure 1 Number of annual adult respiratory cases treated by ECMO and relative survival rates over 15 years (adapted from [73]).

\section{Methods}

To achieve a high standard of reporting, we adopted Preferred Reporting Items for Systematic Reviews and Meta-Analyses (PRISMA) guidelines [20,21].

\section{Search strategy}

The detailed search strategy (identification, screening, eligibility and inclusion process) used to identify relevant studies is summarized in Figure 2. We used a detailed PubMed/MEDLINE, EMBASE and CINAHL query to identify randomized controlled trials (RCTs), controlled observational studies (retrospective and prospective) and case series with $>5$ patients who received extracorporeal gas exchange. Additionally, reference lists from relevant reviews, observational studies and clinical trials were handsearched. Neonatal and pediatric studies (patients $<18$ years of age) were excluded. Language of publication was limited to English and no restriction on the time was set on the primary literature searches. The query was last updated in June 2014.

\section{Study selection}

Two independent researchers (MS and AC) conducted a two-step literature search. Studies were included according to the following criteria: 1) original study published in a peer-reviewed journal; and 2) analyzed/reported the use of extracorporeal gas exchange (i.e., ECMO or pumpless extracorporeal lung assist or extracorporeal $\mathrm{CO}_{2}$ removal) and its specific outcomes for acute respiratory failure in adult patients. Any discrepancies between the two reviewers who examined the titles and abstracts of all relevant citations were resolved by discussion.

\section{Data extraction, quality assessment and analysis}

The two reviewers (MS and $\mathrm{AC}$ ) independently read the entire texts of the retrieved reports and rated study quality using well-established criteria [21,22]. RCT quality was graded using a nine-point scale combining elements from
Jadad's [21] and Chalmers' [23,24] scales, whereas the quality of case-controlled studies was appraised using the Newcastle-Ottawa scale [25]. In addition, both reviewers extracted the following data: First author, year of publication, country, study design, number of patients, demographic data, pre-ECMO ventilation and blood gas data and outcomes. Because of very high heterogeneity between studies, related to different generation ECMO and $\mathrm{ECCO}_{2} \mathrm{R}$ devices used in the last 40 years, different patient populations evaluated, and the scarcity of randomized or quasi-randomized trials (most of which were flawed by major methodological limitations) performed with the latest generation extracorporeal gas exchange techniques, we did not perform meta-analyses of randomized or quasi-randomized trials and choose to report and discuss only crude study results.

\section{Results}

\section{Number of studies selected}

The initial search yielded 535 articles, of which 462 were excluded through title and abstract review, leaving 73 articles potentially meeting our inclusion criteria. After a complete analysis of these, 17 articles were excluded. Of the remaining 56 studies that were evaluated, 4 were RCTs, 7 case-control studies, and 45 case series (Figure 2 and Tables 1, 2, 3, 4, 5 and 6). With the exception of two studies [13,18], all ECMO cohorts had an observational design. Sixteen studies reported on the outcomes of ARDS cases associated with the recent influenza A(H1N1) pandemic (Tables 4 and 5). Ten studies (2 randomized) reporting on $\mathrm{ECCO}_{2} \mathrm{R}$ devices in ARDS patients were retained for the review (Table 6). Overall study validity was adequate, with an average score of 8.1/9.0 on the Newcastle-Ottawa scale appraising the quality of case-control studies.

\section{ECMO and ARDS: studies of historical interest}

In 1968, Kolobow et al. developed the first membrane oxygenator for long-term extracorporeal oxygenation [5]. Three years later, Donald Hill and colleagues described the first use of an ECMO device for acute respiratory failure in humans [4]. They reported on a 24-year-old polytrauma patient, who survived after 75 hours of veno-arterial ECMO. In cohort studies published up to the mid-2000s, the oldest ECMO technology combining roller pump, silicone membrane oxygenator and blood reservoir was used. Survival was $50 \%$ in a cohort of 1,473 patients (1986 to 2006, mean age 34 years, $78 \%$ had VV-ECMO) from the Extracorporeal Life Support Organization (ELSO) registry [26]. In that report, survival was comparable across study periods, although age and severity of disease were significantly higher for the most recent patients. Similarly, Hemmila et al. from Michigan University reported a survival of $52 \%$ in 255 adult patients 


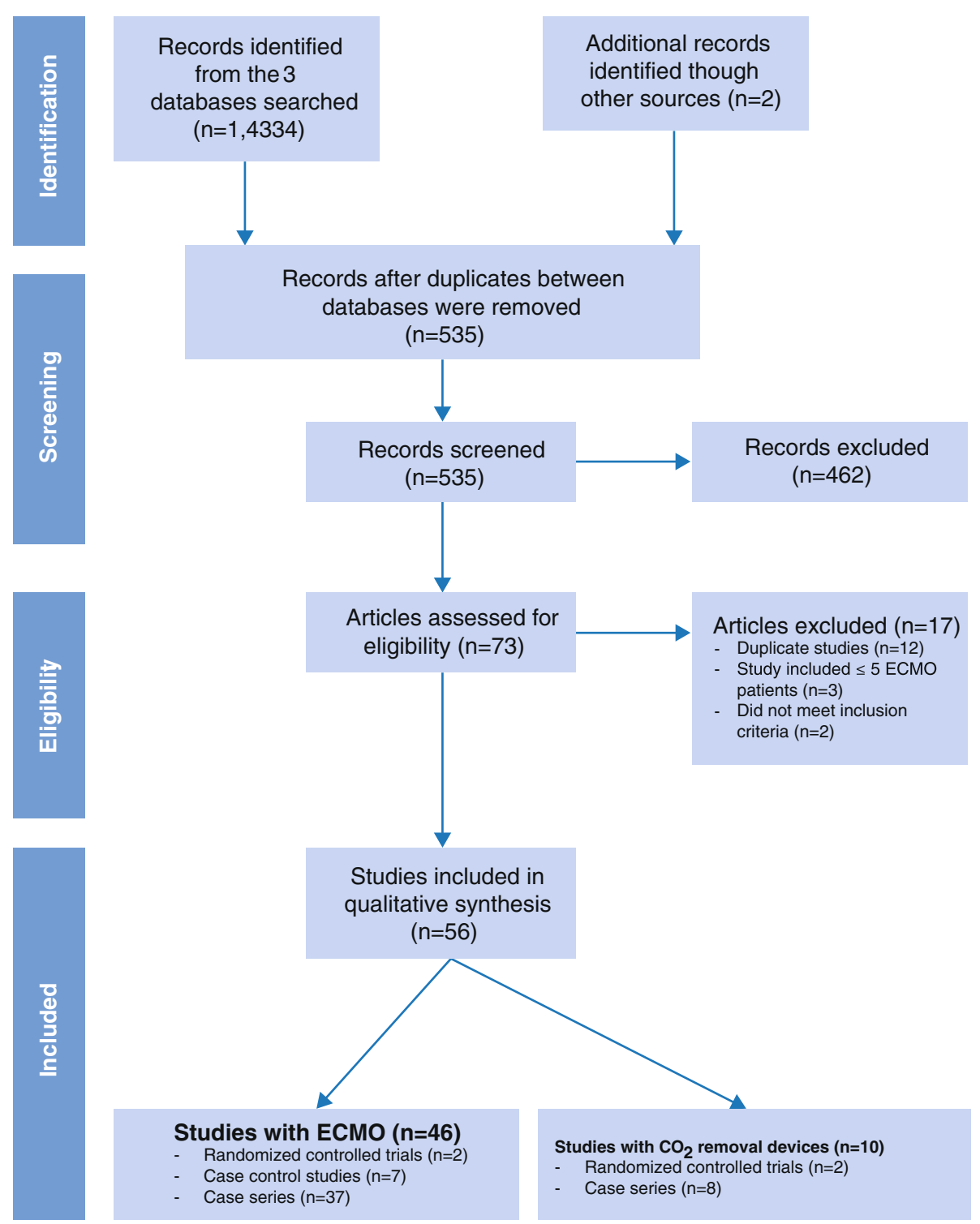

Figure 2 Summary of evidence search and selection: PRISMA flow chart.

treated with ECMO between 1989 and 2003 [27]. Other case-control studies reported similar survival rates for patients treated with ECMO [28,29]. Interestingly, in a cohort of 150 patients with ARDS (mean age 42 years, mean SAPS II 45), of whom 32 received ECMO as a rescue therapy, ECMO support was not independently associated with a higher mortality [30].

\section{ECMO and ARDS: results of randomized controlled trials} Over the last 30 years, only two RCTs of ECMO for ARDS patients have been conducted [13,18]. The National Institutes of Health (NIH) performed the first multicenter trial in the 1970s, enrolling 90 patients with severe ARDS refractory to conventional ventilation [13], of whom 42 received ECMO. Survival was extremely low $(<10 \%)$ and not different between groups. However, that study suffered from major methodological limitations. For example, the mode of ECMO support was only veno-arterial and when no improvement was observed after 5 days, ECMO was removed, which precluded the possibility of late clinical improvement. Because the ECMO group did not receive lung-protective ventilation, severe complications related to barotrauma occurred and since ECMO circuitry was not heparin-coated at that time, a very high percentage of patients had severe hemorrhagic complications due to excessive anticoagulation.

The most recent trial (CESAR), which was conducted in the UK from 2001 to 2006, evaluated a strategy of transfer to a single center (Glenfield, Leicester) that had ECMO capability while the patients randomized to the 
Table 1 Large, recent studies of ECMO for acute respiratory failure: Key patient features

\begin{tabular}{|c|c|c|c|c|c|c|c|c|c|c|c|}
\hline First author [ref] & Design & Prospective & Setting & $\begin{array}{l}\text { Quality } \\
\text { assessment }^{\S}\end{array}$ & Follow up & $\begin{array}{l}\text { ECMO } \\
\text { patients }\end{array}$ & Years & Age & Pneumonia, \% & Mobile ECMO team, \% & SOFA score \\
\hline $\begin{array}{l}\text { Peek, ECMO Arm, } \\
\text { CESAR trial [18] }\end{array}$ & RCT & Y & Multi & RCT6 & 6 months & 68 & $2001-2006$ & $40 \pm 13$ & 62 & 0 & - \\
\hline Schmidt [50] & Case series & Y & Multi & - & Hospital discharge & 2,355 & 2000-2012 & $41(28-54)$ & 34 & - & - \\
\hline Brogan [26] & Case series & $\mathrm{N}$ & Multi & - & Hospital discharge & 1,473 & 1986-2006 & $35(22-53)$ & 26 & - & - \\
\hline Enger [51] & Case series & Y & Single & - & Hospital discharge & 284 & 2008-2013 & $46(43-48)^{\dagger}$ & 49 & 47 & $11(11-12)$ \\
\hline Hemmila [27] & Case series & $\mathrm{N}$ & Single & - & Hospital discharge & 280 & 1989-2003 & $38 \pm 13$ & 31 & 37 & - \\
\hline Schmid [32] & Case series & $\mathrm{N}$ & Single & - & ICU discharge & 176 & 2007-2010 & $48 \pm 17$ & 58 & 34 & $12 \pm 4$ \\
\hline Schmidt [33] & Case series & $N$ & Multi & - & 6 months & 140 & 2008-2012 & $44(30-56)$ & $71^{ \pm}$ & 68 & $12(10-15)$ \\
\hline Lindskov [31] & Case series & Y & Single & - & ICU discharge & 124 & 1977-2011 & $42(16-67)$ & 64 & 85 & - \\
\hline Roch [49] & Case series & N & Single & - & Hospital discharge & 85 & 2009-2013 & $47 \pm 15$ & 86 & 100 & $9(7-11)$ \\
\hline Rega [74] & Case series & $N$ & Single & - & 90 days & 70 & 1997-2005 & $43 \pm 18$ & 41 & - & - \\
\hline Mols [28] & Case-control & Y & Single & 7 & Hospital discharge & 62 & 1991-1999 & $35 \pm 11$ & 58 & 0 & $7(6-9)$ \\
\hline Muller [35] & Case series & Y & Single & - & ICU discharge & 60 & $2006-2008$ & $53(21-78)$ & 42 & 17 & $14(11-16)$ \\
\hline Lewandowski [29] & Case-control & Y & Single & 9 & ICU discharge & 49 & 1989-1995 & $31 \pm 14$ & 37 & - & - \\
\hline Forrest [34] & Case series & $N$ & Multi & - & Hospital discharge & 38 & 2007-2010 & $34(26-42)$ & $89^{\#}$ & 100 & $8(5-10)$ \\
\hline Frenckner [75] & Case series & $N$ & Single & - & ICU & 38 & 1995-2002 & $38(17-61)$ & 60 & 32 & \\
\hline Michaels [46] & Case series & N & Single & - & ICU discharge & 36 & 2009-2012 & $40 \pm 6$ & $58^{\$}$ & 69 & - \\
\hline Beiderlinden [30] & Case-control & Y & Single & 9 & Hospital discharge & 32 & 1998-2003 & $42 \pm 13$ & 53 & - & $14 \pm 3$ \\
\hline
\end{tabular}

$\mathrm{ICU}$, intensive care unit; Multi, multicenter; N, no; RCT, randomized control trial; SOFA; Sequential Organ Failure Assessment; $\mathrm{Y}$, yes.

Data are given as mean \pm SD or median (interquartile range). ${ }^{\ddagger} 26 \%$ were $\mathrm{H} 1 \mathrm{~N} 1$ pneumonia; ${ }^{*} 42 \%$ were $\mathrm{H} 1 \mathrm{~N} 1$ pneumonia; ${ }^{4} 44 \%$ were $\mathrm{H} 1 \mathrm{~N} 1$ pneumonia.

${ }^{5}$ Randomized controlled trial quality was graded using a nine-point scale combining elements from Jadad's [21] and Chalmers' scales [23,24] whereas the validity of case-controlled studies was appraised with the Newcastle-Ottawa scale [25]; ${ }^{+}$in the survivors. 
Table 2 Large, recent studies of ECMO for acute respiratory failure: Key pre-ECMO data and outcomes

\begin{tabular}{|c|c|c|c|c|c|c|c|c|c|c|c|}
\hline $\begin{array}{l}\text { First author } \\
\text { [ref] }\end{array}$ & $\mathrm{PaO}_{2} / \mathrm{FiO}_{2}$ & $\mathrm{pH}$ & $\begin{array}{l}\text { Plateau } \\
\text { pressure }\end{array}$ & PEEP & LIS & $\begin{array}{l}\text { Delay MV - } \\
\text { ECMO, hours }\end{array}$ & $\begin{array}{l}\text { Rescue } \\
\text { therapies, \% }\end{array}$ & $\begin{array}{l}\text { Duration of } \\
\text { ECMO, days }\end{array}$ & Hemorrhage, \% & $\begin{array}{l}\text { Intracerebral } \\
\text { hemorrhage, \% }\end{array}$ & Mortality, n (\%) \\
\hline $\begin{array}{l}\text { Peek, ECMO Arm, } \\
\text { CESAR trial [18] }\end{array}$ & $76 \pm 29$ & $7.1 \pm 0.1$ & - & $14 \pm 9$ & $3.5 \pm 0.6$ & $36(17-104)$ & - & $10(5-23)$ & - & 0 & 33 (37\%) \\
\hline Schmidt [50] & $59(48-75)$ & 7.25 (7.15-7.35) & $36(31-43)^{*}$ & $13(10-16)$ & - & 57 (19-151) & 30 & $7(4-13)$ & - & - & $1,017(43 \%)$ \\
\hline Brogan [26] & $57(45-71)$ & $7.27(7.18-7.36)$ & $40(35-48)^{*}$ & $13(10-16)$ & - & $52(20-160)$ & - & $23 \pm 20$ & 30 & 4 & 732 (50\%) \\
\hline Enger [51] & $69(65-74)^{\dagger}$ & $7.22(7.22-7.25)^{\dagger}$ & $35(34-36)^{*+}$ & $16(16-17)^{\dagger}$ & $3.5(3.4-3.5)^{\dagger}$ & $120(96-168)^{\dagger}$ & - & $10(9-11)^{\dagger}$ & - & - & 117 (41\%) \\
\hline Hemmila [27] & $55 \pm 16$ & $7.31 \pm 0.12$ & $44 \pm 11^{*}$ & $13 \pm 5$ & - & $96 \pm 72$ & - & $9 \pm 8$ & - & 3 & $123(48 \%)$ \\
\hline Schmid [32] & $77 \pm 47$ & $7.2 \pm 0.2$ & $35 \pm 6^{*}$ & $18 \pm 6$ & $3.4 \pm 0.5$ & $144 \pm 240$ & - & $12 \pm 9$ & - & - & $78(44 \%)$ \\
\hline Schmidt [33] & $53(43-60)$ & $7.22(7.15-7.32)$ & $32(30-35)$ & $10(8-12)$ & - & $120(24-264)$ & 94 & $15(8-30)$ & 46 & 3 & $50(36 \%)$ \\
\hline Lindskov [31] & $48(37-60)$ & $7.26 \pm 0.15$ & $37(35-41)$ & - & - & - & - & $9(1-23)$ & - & 9 & $36(29 \%)$ \\
\hline Roch [49] & $60(50-70)$ & $7.1 \pm 0.2$ & $32(29-35)$ & - & $3.5(3.3-3.7)$ & $48(24-194)$ & 85 & $9(7-13)$ & 29 & 2 & $48(56 \%)$ \\
\hline Rega [74] & $56 \pm 18$ & $7.22 \pm 0.18$ & $44 \pm 11^{*}$ & $13 \pm 3$ & - & $108 \pm 178$ & - & $7 \pm 5$ & 20 & - & $40(57 \%)$ \\
\hline Mols [28] & $96 \pm 51$ & $7.30(7.22-7.40)$ & - & - & $3.2 \pm 0.4$ & - & - & $12 \pm 7$ & 7 & 2 & $28(45 \%)$ \\
\hline Muller [35] & $64(48-86)$ & $7.20(7.13-7.30)$ & $36(32-40)^{*}$ & $16(13-20)$ & $3.6(3.3-3.8)$ & $1.0(1.0-4.8)$ & - & $9(5-13)$ & 30 & - & $33(55 \%)$ \\
\hline Lewandowski [29] & - & $7.32 \pm 0.10$ & $39 \pm 7^{*}$ & $12 \pm 3$ & $3.4 \pm 0.2$ & $312 \pm 216$ & - & $23 \pm 17$ & - & - & $22(45 \%)$ \\
\hline Forrest [34] & $57(47-65)$ & $7.20(7.13-7.3)$ & - & $16(12-18)$ & $3.7(3.5-3.7)$ & $48(24-48)$ & 34 & $10(7-17)$ & 37 & 3 & $5(13 \%)$ \\
\hline Frenckner [75] & $47(31-65)$ & - & $41(29-54)^{*}$ & $13(0-20)$ & $3.5(3.0-4.0)$ & $120(24-672)$ & 100 & $17(2-57)$ & 16 & 8 & $13(34 \%)$ \\
\hline Michaels [46] & $52 \pm 3$ & - & - & - & - & $68 \pm 9$ & - & $7 \pm 1$ & - & 6 & $15(40 \%)$ \\
\hline Beiderlinden [30] & $63 \pm 28$ & $7.1 \pm 0.2$ & - & $19 \pm 3$ & $3.8 \pm 0.3$ & $132 \pm 168$ & - & $10(7-15)$ & - & - & 15 (47\%) \\
\hline
\end{tabular}

LIS, lung injury score; MV, mechanical ventilation; PEEP, positive end-expiratory pressure.

Data are given as mean \pm SD or median (interquartile range). ${ }^{*}$ Peak pressure; ${ }^{+}$in the survivors. 
Table 3 Studies of ECMO for ARDS published before 1997 or including < 30 patients

\begin{tabular}{|c|c|c|c|c|c|c|c|c|c|c|c|}
\hline First author [ref] & Design & Prospective & Setting & Follow up & $\begin{array}{l}\text { Quality of } \\
\text { the study }\end{array}$ & $\begin{array}{l}\text { ECMO } \\
\text { patients }\end{array}$ & Years & Age, years & $\begin{array}{l}\text { Pre ECMO } \mathrm{PaO}_{2} / \mathrm{FiO}_{2} \\
\mathrm{mmHg}\end{array}$ & $\begin{array}{l}\text { Length of } \\
\text { ECMO, days }\end{array}$ & Mortality, n (\%) \\
\hline Zapol [13] & $\mathrm{RCT}$ & Y & Multi & ICU discharge & RCT4 & 42 & 1979 & 12 to 65 & $<83$ & $<5$ days & 39 (92\%) \\
\hline Macha [76] & Case series & $\mathrm{N}$ & Single & Hospital discharge & - & 33 & 1990-1995 & $36 \pm 2$ & $59 \pm 5$ & $6 \pm 1$ & $13(39 \%)$ \\
\hline Cordell-Smith [77] & Case series & $N$ & Single & ICU discharge & - & 28 & $1992-2000$ & $27^{*}$ & $62^{*}$ & $6^{*}$ & $8(29 \%)$ \\
\hline Haneya [78] & Case series & Y & Single & ICU discharge & - & 22 & 2010-2011 & $47(36-61)$ & $60(46-75)$ & $13(8-19)$ & $7(32 \%)$ \\
\hline Hodgson [66] & Case series & $N$ & Single & 8 months & - & $21^{ \pm}$ & 2009-2011 & $36 \pm 12$ & $69(50-105)$ & $11(4-16)$ & $3(14 \%)$ \\
\hline Huang [79] & Case series & $N$ & Single & ICU discharge & - & 16 & $2003-2005$ & $32 \pm 22$ & $54 \pm 8$ & $7 \pm 4$ & $6(37 \%)$ \\
\hline Isgrò [80] & Case series & Y & Single & Hospital discharge & - & 12 & 2004-2009 & $35 \pm 19$ & $60 \pm 11$ & - & $6(54 \%)$ \\
\hline Oshima [81] & Case series & $N$ & Single & ICU discharge & - & 11 & 2003-2008 & $52 \pm 24$ & $90 \pm 10$ & $10 \pm 9$ & $5(45 \%)$ \\
\hline Bermudez [82] & Case series & $\mathrm{N}$ & Single & ICU discharge & - & 11 & 2009-2010 & $34(25-54)$ & $45(28-248)$ & $3(0-11)$ & $5(45 \%)$ \\
\hline Goulon [83] & Case series & N & Single & 8 months & - & 11 & 1973-1976 & $29(22-37)$ & $39 \pm 12$ & $3(1-4)$ & $9(82 \%)$ \\
\hline Park [84] & Case series & N & Multi & 60 days & - & 10 & 2011 & $47(14-71)$ & $50(36-56)$ & $5(3-32)$ & $6(60 \%)$ \\
\hline Park [85] & Case series & $\mathrm{N}$ & Single & ICU discharge & - & 9 & 2008-2011 & $56(51-64)$ & $57 \pm 8$ & $12 \pm 6$ & $10(55 \%)$ \\
\hline Huang [79] & Case series & N & Single & 11 (8-51) months & - & 9 & 2004-2007 & $35 \pm 10$ & $49(31-64)$ & $6(3-19)$ & $7(78 \%)$ \\
\hline Rossaint [86] & Case series & $\mathrm{N}$ & Single & Hospital discharge & - & 8 & 1993-1995 & 35 (24-49) & $43 \pm 4$ & $8 \pm 9$ & $2(25 \%)$ \\
\hline
\end{tabular}

ICU: intensive care unit; Multi: multicenter; N: no; RCT: randomized controlled trial; Y: yes.

Data are given as mean \pm SD or median (interquartile range). ${ }^{*}$ Mean; $£ 55 \%$ were H1N1pneumonia. 
Table 4 Large studies of ECMO for H1N1-induced ARDS

\begin{tabular}{|c|c|c|c|c|c|c|c|c|}
\hline & Pham [17] & Noah [16] & Davies [15] & Patroniti [48] & Schmidt [33] & Michaels [46] & Takeda [43] & Holzgraefe [40] \\
\hline Patients, n & $123^{\$}$ & $69^{£}$ & 68 & 49 & 36 & 15 & 14 & 13 \\
\hline Number of centers & 33 & 4 & 15 & 14 & 3 & 1 & 12 & 1 \\
\hline Study design & Case control & Case control & Case control & Case series & Case series & Case series & Case series & Case series \\
\hline Newcastle-Ottawa scale & 9 & 8 & 8 & 7 & - & - & - & - \\
\hline Age, years & $42(32-53)$ & $34(28-46)$ & $36(27-45)$ & $39(32-46)$ & $39(28-53)$ & $34 \pm 4$ & $54(43-60)$ & $31(25-50)$ \\
\hline $\mathrm{BMI}, \mathrm{kg} / \mathrm{m}^{2}$ & $30.5 \pm 8.0$ & - & $29(23-36)$ & $27(24-35)$ & $29(25-36)$ & - & - & $35(31-42)$ \\
\hline Pregnant or postpartum, n (\%) & $18(15)$ & $10(17)$ & $10(16)$ & $4(8)$ & $7(19)$ & $1(7)$ & $1(7)$ & $3(23)$ \\
\hline SOFA & $9.5 \pm 4.0$ & $9(7-10)$ & & $7(6-9)$ & $11(9-14)$ & - & $16(12-19)$ & - \\
\hline Interval MV-ECMO, d & $2(1-5)$ & $4(2-7)$ & $2(1-5)$ & $2(1-5)$ & $2(0-5)$ & $3.5 \pm 0.8$ & $5.0(0.8-8.5)$ & $1(0-7)$ \\
\hline \multicolumn{9}{|l|}{ Pre-ECMO parameters } \\
\hline pH & $7.26 \pm 0.12$ & - & $7.20(7.10-7.30)$ & $7.30(7.22-7.40)$ & $7.22(7.15-7.32)$ & - & - & 7.30(7.30-7.40) \\
\hline $\mathrm{PaO}_{2} / \mathrm{FiO}_{2}, \mathrm{mmHg}$ & $59(51-71)$ & $55(46-63)$ & $56(48-63)$ & $63(56-79)$ & $50(41-55)$ & $62 \pm 6$ & $50(40-55)$ & $52(38-60)$ \\
\hline Plateau pressure, mmHg & $32(29-35)$ & - & $36(33-38)$ & $33(30-35)$ & $32(30-35)$ & - & $30(29-35)$ & $37(31-38)$ \\
\hline Lung injury score & $3.5(3.0-4.0)$ & $3.5(3.5-3.7)$ & $3.8(3.5-4.0)$ & $3.8(3.3-3.8)$ & - & - & - & $3.6(3.3-4.0)$ \\
\hline Any rescue therapy & $91(74)$ & - & - & - & $35(97)$ & - & $4(29)$ & - \\
\hline Nitric oxide, \% & 72 & 19 & 32 & 15 & 89 & - & 7 & - \\
\hline Prone position, $\%$ & 45 & 34 & 20 & 28 & 67 & - & 21 & - \\
\hline Duration of ECMO, days & $11(8-22)$ & $9(6-12)$ & $10(7-15)$ & $10(7-17)$ & $20(9-38)$ & $9 \pm 1$ & $9(4-11)$ & $16(9-30)$ \\
\hline Mortality, n (\%) & $44(36)$ & $22(28)$ & $17(25)$ & $14(29)$ & $6(17)$ & $6(40)$ & $9(64)$ & $2(15)$ \\
\hline
\end{tabular}

BMI: body mass index; SOFA: sequential organ failure assessment; MV: mechanicalventilation.

Data are given as mean \pm SD or median (interquartile range).

${ }_{80} 80$ patients were transferred to Leicester for consideration to receive ECMO and 69 received thedevice. 
Table 5 Studies of ECMO for H1N1-associated ARDS reporting on $\leq 10$ patients

\begin{tabular}{|c|c|c|c|c|c|c|c|c|}
\hline & Roncon [42] & D'Ancona [38] & Roch [41] & Hou [45] & Bonastre [47] & Turner [44] & Chan [37] & Freed [39] \\
\hline Country & Portugal & Italy & France & China & Spain & USA & Hong Kong & Canada \\
\hline Patients, n & 10 & 10 & 9 & 9 & 9 & 7 & 7 & 6 \\
\hline Study design & Case series & Case series & Case series & Case series & Case series & Case series & Case series & Case series \\
\hline Number of centers & 1 & 1 & 1 & 1 & 5 & 1 & 3 & 4 \\
\hline Age, years & $40(36-47)$ & $36(23-55)$ & $49(26-57)$ & $31 \pm 11$ & $36(28-42)$ & $24(16-25)$ & $42(39-50)$ & $33 \pm 7$ \\
\hline $\mathrm{BMI}, \mathrm{kg} / \mathrm{m}^{2}$ & - & $26(21-48)$ & $30(25-80)$ & - & - & $27(26-29)$ & $26(26-27)$ & $33 \pm 7$ \\
\hline Pregnant or postpartum & $1(10)$ & $0(0)$ & $1(11)$ & $4(44)$ & - & $1(14)$ & $0(0)$ & - \\
\hline SOFA & - & - & $9(8-10)$ & - & - & - & - & - \\
\hline Interval MV-ECMO, d & $6.5(2.2-12.2)$ & - & $0.5(0.25-4.0)$ & $6(2-10)$ & $5(2-7)$ & $6.0(1.5-12.5)$ & - & $5.0(2.5-8.3)$ \\
\hline \multicolumn{9}{|l|}{ Pre-ECMO parameter } \\
\hline pH & $7.33(7.28-7.38)$ & - & $7.17(7.04-7.25)$ & - & & - & $7.30(7.19-7.36)$ & $7.31 \pm 0.05$ \\
\hline $\mathrm{PaO}_{2} / \mathrm{FiO}_{2}, \mathrm{mmHg}$ & $69(56-84)$ & - & $52(50-60)$ & $53(45-64)$ & $66(64-102)$ & $57(51-62)$ & $56(53-71)$ & $58 \pm 17$ \\
\hline Plateau pressure, mmHg & $35(32-36)$ & - & $31(30-35)$ & - & - & - & $33(30-35)^{*}$ & $44 \pm 42^{*}$ \\
\hline Lung injury score & $3.5(3.3-3.8)$ & - & $3.6(3.3-3.7)$ & $3.6(3.25-3.75)$ & - & - & $3.8(3.8-3.9)$ & - \\
\hline Any rescue therapy, \% & 70 & 100 & - & - & - & 100 & 14 & 100 \\
\hline Nitric oxide, \% & 60 & - & 67 & - & & 100 & 0 & 67 \\
\hline Prone position, \% & 10 & - & 22 & - & & 0 & 14 & 33 \\
\hline Duration of ECMO, days & $22(14-32)$ & - & $9(4-14)$ & $18(3-90)$ & $6(5-22)$ & $13(8-37)$ & $6(6-10)$ & $15(14-15)$ \\
\hline Mortality, n (\%) & $4(40)$ & $5(50)$ & $5(56)$ & $4(44)$ & $5(56)$ & $2(28)$ & $1(14)$ & $2(33)$ \\
\hline
\end{tabular}

BMI: Body mass index; SOFA: Sequential Organ Failure Assessment; MV: mechanical ventilation

Data are given as mean $\pm S D$ or median (interquartile range). *Peak pressure. 


\section{Table 6 Studies reporting on $\mathrm{ECCO}_{2} \mathrm{R}$ for ARDS}

\begin{tabular}{|c|c|c|c|c|c|c|c|c|c|c|}
\hline & Gattinoni [67] & Morris [14] & Bein [11] & Flörchinger [69] & Brunet [87] & Muellenbach [88] & Nierhaus [89] & Cho [90] & Conrad [91] & Iglesias [68] \\
\hline Country & Italy & USA & Germany & Germany & France & Germany & Germany & Korea & USA & Spain \\
\hline Design & Case series & RCT & RCT & Case series & Case series & Case series & Case series & Case series & Case series & Case series \\
\hline Prospective & Y & Y & Y & Y & Y & $\mathrm{N}$ & $N$ & Y & Y & Y \\
\hline Setting & Single & Mulit & Multi & Single & Single & Single & Single & Single & Multi & Single \\
\hline Quality assessment ${ }^{\S}$ & - & RCT5 & RCT6 & - & - & - & - & - & - & - \\
\hline Type of $\mathrm{CO}_{2}$ removal device, AVM & W & W & AV & AV & W & AV & AV & AV & AV & AV \\
\hline Patients received $\mathrm{ECCO}_{2} \mathrm{R}, \mathrm{n}$ & 43 & 21 & 40 & 159 & 23 & 22 & 13 & 11 & 8 & 7 \\
\hline Years & 1980-1985 & 1987-1991 & $2007-2010$ & 1996-2007 & 1989-1991 & $2002-2006$ & - & 2010 & 1997-1999 & $2005-2006$ \\
\hline Age, years & & & $50 \pm 12$ & $44 \pm 17$ & $29 \pm 10$ & $38 \pm 15$ & $52 \pm 19$ & $58 \pm 15$ & $44 \pm 8$ & $53.7 \pm 16.0$ \\
\hline SOFA score & & & - & 159 & $15 \pm 5$ & - & - & $8.8 \pm 1.8$ & - & - \\
\hline \multicolumn{11}{|l|}{ Pre- $\mathrm{CO}_{2}$ removal } \\
\hline $\mathrm{PO}_{2} / \mathrm{FiO}_{2}, \mathrm{mmHg}$ & 67 & & $152 \pm 37$ & $72 \pm 37$ & $84 \pm 30$ & $61(47-85)^{\$}$ & $100 \pm 29$ & $110 \pm 36.6$ & - & 90 \\
\hline $\mathrm{PCO}_{2}, \mathrm{mmHg}$ & $49 \pm 11$ & & $57 \pm 12$ & $65 \pm 24$ & $56 \pm 20$ & $65(54-72)^{\$}$ & $80 \pm 23$ & $84 \pm 23$ & $90.8 \pm 7.5$ & 70 \\
\hline Plateau pressure, $\mathrm{cmH}_{2} \mathrm{O}$ & - & & $29 \pm 5$ & $37 \pm 6^{*}$ & $51 \pm 9^{*}$ & $40(36-46)^{* \$}$ & $34 \pm 3^{*}$ & $30.1 \pm 7.1$ & - & $22.0 \pm 7.4$ \\
\hline Lung injury score & - & & $2.8 \pm 0.7$ & - & $3.4 \pm 0.4$ & $3.5(3-3.7)$ & - & - & - & $2.9 \pm 0.3$ \\
\hline Delay MV - extracorporeal $\mathrm{CO}_{2}$ removal, days & - & & $<7$ & $7 \pm 13$ & $9.2 \pm 7.7$ & $1(0.5-1.9)$ & $9.4 \pm 10.2$ & $8.6 \pm 12.6$ & - & $4 \pm 2$ \\
\hline Post-cannulation $\mathrm{PCO}_{2}(24 \mathrm{~h}), \mathrm{mmHg}$ & - & & - & $35 \pm 7$ & $41 \pm 7$ & $39(36-42)^{\$}$ & $54 \pm 19$ & $40.7 \pm 10.2$ & $51.8 \pm 3.1$ & 45 \\
\hline Length of $\mathrm{CO}_{2}$ removal device, days & $8 \pm 5$ & & $7.4 \pm 4.0$ & $8 \pm 6$ & $13(1-55)$ & $5.3(3.2-8.2)$ & $12 \pm 22$ & $8.6 \pm 9.4$ & - & $4.3 \pm 2.5$ \\
\hline Serious complication, n (\%) & $3(7)$ & & $3(7)$ & $25(16)$ & $5(22)$ & $5(23)$ & $2(15)$ & $3(27)$ & $0(0)$ & $1(14$ \\
\hline Ischemia lower limb & - & & $1(2)$ & $13(8)$ & - & $3(14)$ & 0 & 0 & 0 & 0 \\
\hline Compartmental syndrome & - & & - & $4(2.5)$ & - & $1(4)$ & 0 & 0 & 0 & 0 \\
\hline Cannula thrombosis & - & & - & $8(5)$ & - & - & 0 & 0 & 0 & 1 \\
\hline Mortality, n (\%) & $22(51)$ & 7 (33) & $7(17)$ & $104(65)$ & $12(52)$ & $6(27)$ & $7(54)$ & $9(82)$ & $4(50)$ & $1(14)$ \\
\hline
\end{tabular}

AV: arteriovenous; W: veno-venous; Multi: multicenter; MV: mechanical ventilation; N: no; RCT: randomized controlled trial; SOFA: Sequential Organ Failure Assessment; Y: yes; ${ }^{\text {*eak }}$ pressure; ${ }^{5}$ RCT quality was graded using a nine-point scale combining elements from Jadad's [21] and Chalmers' scales [23,24] whereas the validity of case-controlled studies was appraised with the Newcastle-Ottawa scale [25]. Data are given as mean \pm SD or median (interquartile range). 
control group were treated conventionally at designated treatment centers [18]. The primary endpoint combining mortality or severe disability 6 months after randomization was lower for the 90 patients randomized to the ECMO group ( $37 \%$ vs. $53 \%, \mathrm{p}=0.03$ ). However, the results of that trial should be analyzed carefully. First, 22 patients randomized to the ECMO arm did not receive ECMO (died before or during transport, improved with conventional management at the referral center or had a contraindication to heparin). Second, no standardized protocol for lung-protective mechanical ventilation existed in the control group and the time spent receiving 'protective' mechanical ventilation was significantly higher in the ECMO arm. Third, more patients received corticosteroids in the ECMO group.

\section{ECMO and ARDS: retrospective series using the latest technology}

In the most recent series, patients benefited from the latest ECMO technology, which includes a centrifugal pump, a polymethylpentene membrane oxygenator and tubing with biocompatible surface treatment. Mortality rates range from 36 to $56 \%$ in the studies performed in the last 15 years and reporting outcomes of $>30$ ECMO patients (Tables 1 and 2). Interestingly, ECMO was provided through a mobile ECMO rescue team in some of these studies. For example, in a series of 124 patients treated at a Danish center between 1997 and 2011 [31], survival was $71 \%$ and $85 \%$ of these patients received ECMO via a mobile unit before being transferred to the referral hospital. Similarly, in the Regensburg cohort, 59/176 received ECMO at another hospital by a mobile unit [32]. In a multicenter French cohort of 140 patients treated between 2008 and 2012, 68\% patients were retrieved via a mobile ECMO team and their prognosis was comparable to those who received VV-ECMO support in their initial hospital [33].

ECMO support may also cause frequent, severe and potentially life-threatening complications (Table 2), such as bleeding, infections, intravascular hemolysis, thrombocytopenia or consumption coagulopathy [26,33-36].

\section{Results of ECMO for pandemic influenza A (H1N1)-associated ARDS}

Mortality rates ranged from 14 to $64 \%$ in the 16 studies from 11 countries reporting on the experience of ECMO for influenza $\mathrm{A}(\mathrm{H} 1 \mathrm{~N} 1)$-associated ARDS (Tables 4 and 5) $[15-17,33,37-48]$. The Australia and New Zealand collaborative group (ANZICS) was the first to report its experience [15]. Despite extreme disease severity at the time of ECMO initiation (median $\mathrm{PaO}_{2} / \mathrm{FiO}_{2}$ ratio 56 $\mathrm{mmHg}$, median positive end-expiratory pressure [PEEP] $18 \mathrm{cmH}_{2} \mathrm{O}$ and median lung injury score [LIS] of 3.8), only $25 \%$ of the 68 ECMO patients died. A British collaborative cohort series [16] depicted the outcome of 80 patients transferred into ECMO referral centers in the United Kingdom of whom 69 received ECMO. Mortality in this cohort was $27.5 \%$. A propensity-matched analysis comparing survival of patients referred for consideration of ECMO to other ARDS patients showed better outcomes for referred patients. By contrast, mortality of propensitymatched patients treated conventionally was comparable to that of ECMO patients in French ICUs of the REVA network. However, only 50\% of ECMO patients were successfully matched with control ARDS patients, while unmatched ECMO patients were younger, suffered more severe respiratory failure and had considerably lower mortality [17]. Interestingly, a higher plateau pressure under ECMO was independently associated with mortality, indicating for the first time that an ultraprotective ventilation strategy with reduction of plateau pressure to around $25 \mathrm{cmH}_{2} \mathrm{O}$ following ECMO installation might improve outcomes. Lastly, mortality was $29 \%$ in a cohort of 49 proven influenza $\mathrm{A}(\mathrm{H} 1 \mathrm{~N} 1)$ patients from the $14 \mathrm{ECMO}$ centers of the ECMO-NET Italian collaborative group [48]. In this series, patients ventilated for less than 7 days before ECMO initiation had a significantly higher survival.

\section{Mortality risk factors and outcome prediction for ECMO candidates}

Factors associated with poor outcomes after ECMO for acute respiratory failure include older age $[26,27,30,32,33,49,50]$, a greater number of days of mechanical ventilation before ECMO establishment [26,27,30,33,50], a higher number of organ failures $[26,27,30,32,33,49,50]$, low preECMO respiratory system compliance [50], and immunosuppression [33,50,51]. Predictive survival models have been recently developed that might help clinicians select appropriate candidates for ECMO [33,49-52]. For example, the Respiratory Extracorporeal Membrane Oxygenation Survival (RESP) score [50], constructed on data extracted from a large multicenter international population $(\mathrm{n}=2,355)$, computes 12 simple pre-ECMO parameters, to provide a relevant and validated tool predicting survival after ECMO for acute respiratory failure. Cumulative predicted hospital survival rates were 92, 76, 57, 33 and $18 \%$ for five RESP-score risk classes, I ( $\geq 6$ ), II (3 to 5), III ( -1 to 2$)$, IV ( -5 to -2 ) and V ( $\leq-6)$, respectively.

\section{Volume-outcome effect and ECMO activity organization}

Recent analyses of large pediatric databases have suggested a significant relationship between the volume of patients treated by center and ECMO patient prognosis [53-55]. ECMO case-series published after the pandemic influenza A(H1N1) might also allow a comparative analysis of worldwide results obtained for a very homogeneous disease (Tables 4 and 5). These data suggest that the 
best results were obtained for patients managed in expert centers treating a sufficient number of patients and in countries where ECMO activity was organized and regulated, as was the case in the United Kingdom [56], Italy [57] and in Australia and New Zealand [58]. A recent position paper [59] by an international group of physicians with expertise in ECMO for severe respiratory failure advocated for regional and interregional organization of ECMO activity through networks of hospitals around an ECMO referral center with a mobile ECMO unit $[34,60,61]$ to retrieve the most severe ARDS patients. This group also suggested that at least 20 ECMO cases should be performed per year at each referral center [59]. Furthermore, high volume and expert referral centers may provide better prevention and management of the severe complications that can occur during long ECMO runs (Table 2).

\section{Long term outcomes after ECMO}

Durations of intensive care and hospital stays of ECMO patients are long and frequently exceed one month [26], [33]. Thus, evaluation of the impact of such complex therapy on long-term pulmonary function, quality of life and psychological status appears crucial in the decision process to use ECMO in ARDS patients. To date, long-term prognosis after ECMO for ARDS has rarely been evaluated. Linden et al. reported long-term outcomes of 21 ARDS survivors rescued with ECMO [62]. In this study, most of the patients had limited fibrosis lesions on $\mathrm{CT}$ scan. Respiratory function tests were within normal limits. However, patients reported deterioration in pulmonary symptoms measured by the St George Respiratory Questionnaire, although these symptoms were comparable to those reported in other series of ARDS patients treated conventionally. Similarly, patients in the ECMO arm of the CESAR trial [18] exhibited comparable or better health-related quality of life scores (measured by the SF-36 questionnaire) than those reported by patients with ARDS treated with conventional management $[63,64]$. Exertional dyspnea was reported by $50 \%$ and $40 \%$ of 12 influenza A(H1N1) ECMO patients and 25 controls, respectively [65]. Anxiety and depressive symptoms were reported by $50 \%$ and $28 \%$ of ECMO patients, respectively, whereas $41 \%$ were at risk of post-traumatic stress disorder (PTSD) [65]. By contrast, results of the Melbourne group were poorer, with only $26 \%$ of long-term survivors having returned to their previous work at eight-month-follow-up [66]. Similar to previous studies, mean SF-36 scores in the ECMO population were lower than these previously described with ARDS survivors in the domains of general health, mental health, vitality and social function. Lastly, the largest study published to date was reported by Schmidt et al. [33] on a population of 84 -month survivors. In that series, $36 \%$ of the patients reported exertional dyspnea, whereas $30 \%$ were still receiving pulmonary treatments after a median 17-month follow-up. Health-related quality of life evaluation in $80 \%$ of the 6 -month survivors revealed satisfactory mental health but persistent physical and emotional-related difficulties, with anxiety, depression or PTSD symptoms reported by 34,25 and $16 \%$, respectively.

\section{Results of extracorporeal $\mathrm{CO}_{2}$ removal techniques for ARDS patients}

To date, studies on $\mathrm{ECCO}_{2} \mathrm{R}$ in ARDS patients are scarce and mostly small retrospective case series (Table 6). Gattinoni et al. reported in 1986 the first cohort of 43 patients with severe ARDS treated with veno-venous, low flow $(200-300 \mathrm{ml} / \mathrm{min}) \mathrm{ECCO}_{2} \mathrm{R}$, which needed a boot volume of almost two liters of blood [67]. In this series, $\mathrm{ECCO}_{2} \mathrm{R}$ duration was 5 days, daily blood losses were large (> 1,800 ml/24 hours) and survival was 49\%. A randomized study using the same technology was carried out in the early 1990s by Morris et al. [14]. It was stopped for futility after the enrolment of only 40 patients and mortality was $67 \%$ in the 21 patients randomized to $\mathrm{ECCO}_{2} \mathrm{R}$. In the 2000s, case series, which used the pumpless arteriovenous shunt (extracorporeal interventional lung assist, iLA, Novalung, Heilbronn, Germany) were published. Iglesias et al. [68] reported the outcome of seven patients with ARDS after pneumonectomy. The $\mathrm{ECCO}_{2} \mathrm{R}$ device was left in place for four days, $\mathrm{CO}_{2}$ removal was $255 \mathrm{ml} / \mathrm{min}$ allowing significant reduction in tidal volume and 6/7 patients survived (Table 6). In a larger German cohort of 156 patients, a higher mortality was reported (65\%). Of note, $16 \%$ of the patients experienced serious complications in that cohort, particularly leg ischemia related to femoral arterial cannulation and need for higher dose catecholamines (Table 6) [69].

The concept of ultraprotective mechanical ventilation was tested in a proof-of concept trial, with $\mathrm{CO}_{2}$ removal performed by a modified veno-venous hemofiltration platform. In 10 patients with plateau pressure of 28-30 $\mathrm{cmH}_{2} \mathrm{O}$ at baseline, $\mathrm{ECCO}_{2} \mathrm{R}$ allowed a reduction of tidal volume (from 6 to $4 \mathrm{ml} / \mathrm{kg}$ ) and of plateau pressure (from 29 to $25 \mathrm{cmH}_{2} \mathrm{O}$ ), while maintaining $\mathrm{PaCO}_{2}$ around $50 \mathrm{mmHg}$ [19]. This protective ventilation strategy was also associated with a significant reduction in proinflammatory cytokine levels in bronchoalveolar (BAL) fluid. This ultraprotective ventilation strategy was recently evaluated in the Xtravent trial [11], which randomized 79 patients to conventional mechanical ventilation using the ARDSNet strategy [70] or to tidal volume reduction to 3 $\mathrm{ml} / \mathrm{kg}$ permitted by $\mathrm{CO}_{2}$ removal with the Novalung AV pumpless $\mathrm{ECCO}_{2} \mathrm{R}$ device. The numbers of ventilator-free days at day 60 were not different between groups. However, a post-hoc subgroup analysis revealed that 
patients with lower $\mathrm{PaO}_{2} / \mathrm{FiO}_{2}(\leq 150 \mathrm{mmHg}$ ) at randomization had significantly more ventilator-free days at days 28 and 60 and were more rapidly weaned from mechanical ventilation.

\section{Conclusion and perspectives}

We report the results of 56 studies (including 4 RCTs) evaluating extracorporeal gas exchange techniques (ECMO or $\mathrm{ECCO}_{2}-\mathrm{R}$ ) to treat moderate to severe acute respiratory failure in adult patients. Major heterogeneity in study populations, disease severity, type of device used and time at which studies were performed creates insuperable hurdles to design relevant meta-analyses. Results of the most recent randomized CESAR trial, which was conducted in the UK from 2001 to 2006, suggested that a strategy of transfer to an ECMO referral center for consideration to receive ECMO was associated with better outcomes. However, that trial was highly criticized for methodological limitations. Additionally, non-randomized case-series of ECMO, including propensity-matched case-control studies, are prone to important selection biases weakening interpretation of their results. Although early implementation of VV-ECMO in severe ARDS patients might allow significant reduction in ventilatorinduced lung injury (VILI) and may rescue patients dying of refractory hypoxemia, more evidence is urgently needed to evaluate the actual impact of the technique on patientcentered outcomes compared to optimization of conventional treatments, including prone positioning [2]. This is the main objective of the ongoing international multicenter randomized Extracorporeal Membrane Oxygenation for Severe Acute Respiratory Distress Syndrome (EOLIA) trial (ClinicalTrials.gov Identifier: NCT01470703), which will test the efficacy of early VV-ECMO in patients with severe ARDS with tight control of mechanical ventilation in the control group, initiation of ECMO prior to transportation to ECMO centers, and the use of ECMO in every patient randomly assigned to receive it [71].

Pathophysiological, experimental and clinical data suggest that an 'ultraprotective' mechanical ventilation strategy reducing tidal volume to $3-4 \mathrm{ml} / \mathrm{kg}$ predicted body weight and plateau pressure to $<25 \mathrm{cmH}_{2} \mathrm{O}$ may further reduce VILI and ARDS-associated morbidity and mortality in less severe ARDS patients. Hypercapnia induced by tidal volume reduction in this setting might be efficiently controlled by the latest generation low-flow, venovenous $\mathrm{ECCO}_{2} \mathrm{R}$ devices, which are more efficient, more biocompatible and associated with fewer hemorrhagic complications because they require less anticoagulation than devices evaluated in the 1980s and 90s, which did not achieve significant mortality reduction. However, the uncritical and large adoption of this strategy is premature and problematic without rigorous evaluation of associated risks and benefits. This will be the objective of the large randomized Strategy of Ultra Protective lung ventilation with Extracorporeal $\mathrm{CO}_{2}$ Removal for New-Onset moderate to severe ARDS (SUPERNOVA) trial, which will test the benefits of early tidal volume and plateau pressure reduction allowed by the latest generation $\mathrm{ECCO}_{2} \mathrm{R}$ device in patients with moderate forms of ARDS [72].

Lastly, future studies of extracorporeal gas exchange should also include detailed evaluation of physical and psychosocial rehabilitation that could lead to improved long-term health-related quality of life in this population of patients.

\section{Abbreviations}

ARDS: Acute respiratory distress syndrome; AV: Arteriovenous; BMI: Body mass index; $\mathrm{ECCO}_{2} \mathrm{R}$ : Extracorporeal carbon dioxide removal;

ECMO: Extracorporeal membrane oxygenation; ELSO: Extracorporeal life support organization; ICU: Intensive care unit; LIS: Lung injury score; MV: Mechanical ventilation; PEEP: Positive end-expiratory pressure; PRISMA: Preferred reporting items for systematic reviews and meta-analyses; RCT: Randomized control trial; RESP: Respiratory extracorporeal membrane oxygenation survival; SOFA: Sequential organ failure assessment; VILI: Ventilator-induced lung injury; W: Veno-venous.

\section{Competing interests}

Pr Combes is the primary investigator of the EOLIA trial, NCT01470703, a randomized trial of W-ECMO supported in part by MAQUET. Pr Combes has received honoraria for lectures by MAQUET, BAXTER AND ALung. The other authors declare that they have no competing interests.

\section{Acknowledgement}

Matthieu Schmidt was supported by the French Intensive Care Society (SRLF), the "Fonds de dotation Recherche en Santé Respiratoire, 2012", the "Collège des Enseignants de Réanimation Médicale" and the "Département du Développement professionnel continu médical - Assistance publiqueHôpitaux de Paris".

\section{Declarations}

The publication charges for this article were funded by the corresponding author's institution.

\section{Author details}

${ }^{1}$ Université Pierre et Marie Curie, Medical-Surgical Intensive Care Unit, iCAN, Institute of Cardiometabolism and Nutrition, Hôpital de la Pitié-Salpêtrière, Assistance Publique-Hôpitaux de Paris, Paris, France. ${ }^{2}$ The Australian \& New Zealand Intensive Care Research Centre and the Intensive Care Department, Alfred Hospital, Melbourne, Australia.

Published online: 16 March 2015

\section{References}

1. Dreyfuss D, Soler P, Basset G, Saumon G. High inflation pressure pulmonary edema. Respective effects of high airway pressure, high tidal volume, and positive end-expiratory pressure. Am Rev Respir Dis. 1988;137:1159-64.

2. Guerin C, Reignier J, Richard JC, et al. Prone positioning in severe acute respiratory distress syndrome. N Engl J Med. 2013;368:2159-68.

3. Papazian L, Forel JM, Gacouin A, et al. Neuromuscular blockers in early acute respiratory distress syndrome. N Engl J Med. 2010;363:1107-16.

4. Hill JD, O'Brien TG, Murray JJ, et al. Prolonged extracorporeal oxygenation for acute post-traumatic respiratory failure (shock-lung syndrome). Use of the Bramson membrane lung. N Engl J Med. 1972;286:629-34.

5. Kolobow T, Zapol W, Pierce JE, Keeley AF, Replogle RL, Haller A. Partial extracorporeal gas exchange in alert newborn lambs with a membrane artificial lung perfused via an A-V shunt for periods up to 96 hours. Trans Am Soc Artif Intern Organs. 1968;14:328-34.

6. Combes A, Brechot N, Luyt CE, Schmidt M. What is the niche for extracorporeal membrane oxygenation in severe acute respiratory distress syndrome? Curr Opin Crit Care. 2012;18:527-32. 
7. Brodie D, Bacchetta M. Extracorporeal membrane oxygenation for ARDS in adults. N Engl J Med. 2012;365:1905-14.

8. Combes A, Bacchetta M, Brodie D, Muller T, Pellegrino V. Extracorporeal membrane oxygenation for respiratory failure in adults. Curr Opin Crit Care. 2012;18:99-104.

9. MacLaren G, Combes A, Bartlett RH. Contemporary extracorporeal membrane oxygenation for adult respiratory failure: life support in the new era. Intensive Care Med. 2012;38:210-20

10. Cove ME, Maclaren G, Federspiel WJ, Kellum JA. Bench to bedside review: extracorporeal carbon dioxide removal, past present and future. Crit Care. 2012;16:232

11. Bein T, Weber-Carstens S, Goldmann A, et al. Lower tidal volume strategy (approximately $3 \mathrm{ml} / \mathrm{kg}$ ) combined with extracorporeal $\mathrm{CO} 2$ removal versus 'conventional' protective ventilation $(6 \mathrm{ml} / \mathrm{kg})$ in severe ARDS: the prospective randomized Xtravent-study. Intensive Care Med. 2013;39:847-56.

12. Schmidt M, Tachon G, Devilliers C, et al. Blood oxygenation and decarboxylation determinants during venovenous ECMO for respiratory failure in adults. Intensive Care Med. 2013;39:838-46.

13. Zapol WM, Snider MT, Hill JD, et al. Extracorporeal membrane oxygenation in severe acute respiratory failure. A randomized prospective study. JAMA. 1979;242:2193-6.

14. Morris AH, Wallace CJ, Menlove RL, et al. Randomized clinical trial of pressure-controlled inverse ratio ventilation and extracorporeal $\mathrm{CO} 2$ removal for adult respiratory distress syndrome. Am J Respir Crit Care Med. 1994;149:295-305.

15. Davies A, Jones D, Bailey M, et al. Extracorporeal Membrane Oxygenation for 2009 Influenza A(H1N1) Acute Respiratory Distress Syndrome. JAMA. 2009;302:1888-95.

16. Noah MA, Peek GJ, Finney SJ, et al. Referral to an extracorporeal membrane oxygenation center and mortality among patients with severe 2009 influenza A(H1N1). JAMA. 2011;306:1659-68.

17. Pham $\mathrm{T}$, Combes $\mathrm{A}$, Roze $\mathrm{H}$, et al. Extracorporeal membrane oxygenation for pandemic influenza $A(\mathrm{H} 1 \mathrm{~N} 1)$-induced acute respiratory distress syndrome: A cohort study and propensity-matched analysis. Am J Respir Crit Care Med. 2013;187:276-85.

18. Peek GJ, Mugford M, Tiruvoipati R, et al. Efficacy and economic assessment of conventional ventilatory support versus extracorporeal membrane oxygenation for severe adult respiratory failure (CESAR): a multicentre randomised controlled trial. Lancet. 2009;374:1351-63.

19. Terragni PP, Del Sorbo L, Mascia L, et al. Tidal volume lower than $6 \mathrm{ml} / \mathrm{kg}$ enhances lung protection: role of extracorporeal carbon dioxide removal. Anesthesiology. 2009;111:826-35.

20. Moher D, Liberati A, Tetzlaff J, Altman DG. Preferred reporting items for systematic reviews and meta-analyses: the PRISMA statement. Ann Intern Med. 2009:151:264-9.

21. Jadad AR, Moore RA, Carroll D, et al. Assessing the quality of reports of randomized clinical trials: is blinding necessary? Control Clin Trials. 1996;17:1-12.

22. Wells GA, Shea B, O'Connell D et al. The Newcastle-Ottawa Scale (NOS) for assessing the quality of nonrandomised studies in meta-analyses. http://www.ohri.ca/programs/clinical_epidemiology/oxford.asp.

23. Chalmers TC, Smith Jr H, Blackburn B, et al. A method for assessing the quality of a randomized control trial. Control Clin Trials. 1981;2:31-49.

24. Mitchell MD, Mikkelsen ME, Umscheid CA, Lee I, Fuchs BD, Halpern SD. A systematic review to inform institutional decisions about the use of extracorporeal membrane oxygenation during the H1N1 influenza pandemic. Crit Care Med. 2010:38:1398-404.

25. Stang A. Critical evaluation of the Newcastle-Ottawa scale for the assessment of the quality of nonrandomized studies in meta-analyses. Eur J Epidemiol. 2010;25:603-5

26. Brogan TV, Thiagarajan RR, Rycus PT, Bartlett RH, Bratton SL. Extracorporeal membrane oxygenation in adults with severe respiratory failure: a multi-center database. Intensive Care Med. 2009;35:2105-14.

27. Hemmila MR, Rowe SA, Boules TN, et al. Extracorporeal life support for severe acute respiratory distress syndrome in adults. Ann Surg. 2004:240:595-605.

28. Mols G, Loop T, Geiger K, Farthmann E, Benzing A. Extracorporeal membrane oxygenation: a ten-year experience. Am J Surg. 2000;180:144-54.

29. Lewandowski $K$, Rossaint $R$, Pappert $D$, et al. High survival rate in 122 ARDS patients managed according to a clinical algorithm including extracorporeal membrane oxygenation. Intensive Care Med. 1997;23:819-35.
30. Beiderlinden M, Eikermann M, Boes T, Breitfeld C, Peters J. Treatment of severe acute respiratory distress syndrome: role of extracorporeal gas exchange. Intensive Care Med. 2006;32:1627-31.

31. Lindskov C, Jensen RH, Sprogoe P, et al. Extracorporeal membrane oxygenation in adult patients with severe acute respiratory failure. Acta Anaesthesiol Scand. 2013;57:303-11.

32. Schmid C, Philipp A, Hilker M, et al. Venovenous extracorporeal membrane oxygenation for acute lung failure in adults. J Heart Lung Transplant. 2012;31:9-15.

33. Schmidt M, Zogheib E, Roze $\mathrm{H}$, et al. The PRESERVE mortality risk score and analysis of long-term outcomes after extracorporeal membrane oxygenation for severe acute respiratory distress syndrome. Intensive Care Med. 2013;39:1704-13.

34. Forrest P, Ratchford J, Burns B, et al. Retrieval of critically ill adults using extracorporeal membrane oxygenation: an Australian experience. Intensive Care Med. 2011;37:824-30

35. Muller T, Philipp A, Luchner A, et al. A new miniaturized system for extracorporeal membrane oxygenation in adult respiratory failure. Crit Care. 2009;13:R205.

36. Schmidt M, Brechot N, Hariri S, et al. Nosocomial infections in adult cardiogenic shock patients supported by venoarterial extracorporeal membrane oxygenation. Clin Infect Dis. 2012;55:1633-41.

37. Chan KK, Lee KL, Lam PK, Law Kl, Joynt GM, Yan WW. Hong Kong's experience on the use of extracorporeal membrane oxygenation for the treatment of influenza A (H1N1). Hong Kong Med J. 2010;16:447-54.

38. D'Ancona G, Capitanio G, Chiaramonte G, et al. Extracorporeal membrane oxygenator rescue and airborne transportation of patients with influenza $\mathrm{A}$ ( $\mathrm{H} 1 \mathrm{~N} 1)$ acute respiratory distress syndrome in a Mediterranean underserved area. Interact Cardiovasc Thorac Surg. 2011;12:935-7.

39. Freed DH, Henzler D, White CW, et al. Extracorporeal lung support for patients who had severe respiratory failure secondary to influenza A (H1N1) 2009 infection in Canada. Can J Anaesth. 2010;57:240-7.

40. Holzgraefe B, Broome M, Kalzen H, Konrad D, Palmer K, Frenckner B. Extracorporeal membrane oxygenation for pandemic H1N1 2009 respiratory failure. Minerva Anestesiol. 2010;76:1043-51.

41. Roch A, Lepaul-Ercole R, Grisoli D, et al. Extracorporeal membrane oxygenation for severe influenza A (H1N1) acute respiratory distress syndrome: a prospective observational comparative study. Intensive Care Med. 2010;36:1899-905.

42. Roncon-Albuquerque Jr R, Basilio C, Fiqueiredo P, et al. Portable miniaturized extracorporeal membrane oxygenation systems for H1N1-related severe acute respiratory distress syndrome: A case series. J Crit Care. 2012;27:454-63.

43. Takeda S, Kotani T, Nakagawa $\mathrm{S}$, et al. Extracorporeal membrane oxygenation for 2009 influenza $A(H 1 N 1)$ severe respiratory failure in Japan. J Anesth. 2012;26:650-7.

44. Turner DA, Rehder KJ, Peterson-Carmichael SL, et al. Extracorporeal membrane oxygenation for severe refractory respiratory failure secondary to 2009 H1N1 influenza A. Respir Care. 2011:56:941-6.

45. Hou X, Guo L, Zhan Q, et al. Extracorporeal membrane oxygenation for critically ill patients with 2009 influenza A (H1N1)-related acute respiratory distress syndrome: preliminary experience from a single center. Artif Organs. 2012:36:780-6.

46. Michaels AJ, Hill JG, Bliss D, et al. Pandemic flu and the sudden demand for ECMO resources: a mature trauma program can provide surge capacity in acute critical care crises. J Trauma Acute Care Surg. 2013;74:1493-7.

47. Bonastre J, Suberviola B, Pozo JC, et al. Extracorporeal lung support in patients with severe respiratory failure secondary to the 2010-2011 winter seasonal outbreak of influenza A (H1N1) in Spain. Med Intensiva. 2012:36:193-9

48. Patroniti N, Zangrillo A, Pappalardo F, et al. The Italian ECMO network experience during the 2009 influenza $A(\mathrm{H} 1 \mathrm{~N} 1)$ pandemic: preparation for severe respiratory emergency outbreaks. Intensive Care Med. 2011;37:1447-57

49. Roch A, Hraiech $S$, Masson E, et al. Outcome of acute respiratory distress syndrome patients treated with extracorporeal membrane oxygenation and brought to a referral center. Intensive Care Med. 2014;40:74-83.

50. Schmidt M, Bailey M, Sheldrake J, et al. Predicting survival after extracorporeal membrane oxygenation for severe acute respiratory failure. The Respiratory Extracorporeal Membrane Oxygenation Survival Prediction (RESP) Score. Am J Respir Crit Care Med. 2014;189:1374-82. 
51. Enger T, Philipp A, Videm $V$, et al. Prediction of mortality in adult patients with severe acute lung failure receiving veno-venous extracorporeal membrane oxygenation: a prospective observational study. Crit Care. 2014;18:R67.

52. Pappalardo F, Pieri M, Greco T, et al. Predicting mortality risk in patients undergoing venovenous ECMO for ARDS due to influenza A (H1N1) pneumonia: the ECMOnet score. Intensive Care Med. 2013;39:275-81.

53. Campbell BT, Braun TM, Schumacher RE, Bartlett RH, Hirschl RB. Impact of ECMO on neonatal mortality in Michigan (1980-1999). J Pediatr Surg. 2003;38:290-5

54. Jen HC, Shew SB. Hospital readmissions and survival after nonneonatal pediatric ECMO. Pediatrics. 2010;125:1217-23.

55. Karamlou T, Vafaeezadeh M, Parrish AM, et al. Increased extracorporeal membrane oxygenation center case volume is associated with improved extracorporeal membrane oxygenation survival among pediatric patients. J Thorac Cardiovasc Surg. 2013;145:470-5.

56. Extracorporeal membrane oxygenation for severe acute respiratory failure in adults. NHS NICE GUIDANCE Available at: http://www.nice.org.uk/guidance/ IPG391. Accessed December 2014

57. ECMOnet. Available at: http://www.ecmonet.org/. Accessed March 14th 2014.

58. NSW Critical Care Tertiary Referral Networks and Transfer of Care (ADULTS). Available at: http://www0.health.nsw.gov.au/policies/pd/2010/pdf/ PD2010_021.pdf. Accessed December 14, 2014

59. Combes A, Brodie D, Bartlett R, et al. Position paper for the organization of extracorporeal membrane oxygenation programs for acute respiratory failure in adult patients. Am J Respir Crit Care Med. 2014;190:488-96.

60. Beurtheret S, Mordant P, Paoletti X, et al. Emergency circulatory support in refractory cardiogenic shock patients in remote institutions: a pilot study (the cardiac-RESCUE program). Eur Heart J. 2013;34:112-20.

61. Linden V, Palmer K, Reinhard J, et al. High survival in adult patients with acute respiratory distress syndrome treated by extracorporeal membrane oxygenation, minimal sedation, and pressure supported ventilation. Intensive Care Med. 2000;26:1630-7.

62. Linden VB, Lidegran MK, Frisen G, Dahlgren P, Frenckner BP, Larsen F. ECMO in ARDS: a long-term follow-up study regarding pulmonary morphology and function and health-related quality of life. Acta Anaesthesiol Scand. 2009;53:489-95.

63. Herridge MS, Cheung AM, Tansey CM, et al. One-year outcomes in survivors of the acute respiratory distress syndrome. N Engl J Med. 2003;348:683-93.

64. Schelling G, Stoll C, Haller M, et al. Health-related quality of life and posttraumatic stress disorder in survivors of the acute respiratory distress syndrome. Crit Care Med. 1998;26:651-9.

65. Luyt $\mathrm{CE}$, Combes $\mathrm{A}$, Becquemin $\mathrm{MH}$, et al. Long-term outcomes of pandemic 2009 influenza A(H1N1)-associated severe ARDS. Chest. 2012;142:583-92.

66. Hodgson $\mathrm{CL}$, Hayes $\mathrm{K}$, Everard $\mathrm{T}$, et al. Long-term quality of life in patients with acute respiratory distress syndrome requiring extracorporeal membrane oxygenation for refractory hypoxaemia. Crit Care. 2012;16:R202.

67. Gattinoni L, Pesenti A, Mascheroni D, et al. Low-frequency positive-pressure ventilation with extracorporeal $\mathrm{CO} 2$ removal in severe acute respiratory failure. JAMA. 1986;256:881-6.

68. Iglesias M, Martinez E, Badia JR, Macchiarini P. Extrapulmonary ventilation for unresponsive severe acute respiratory distress syndrome after pulmonary resection. Ann Thorac Surg. 2008;85:237-44.

69. Florchinger B, Philipp A, Klose A, et al. Pumpless extracorporeal lung assist: a 10-year institutional experience. Ann Thorac Surg. 2008;86:410-7 (discussion 417).

70. The Acute Respiratory Distress Syndrome Network. Ventilation with lower tidal volumes as compared with traditional tidal volumes for acute lung injury and the acute respiratory distress syndrome. N Engl J Med. 2000;342:1301-8.

71. EOLIA trial. Available at: http://www.clinicaltrials.gov/ct2/show/ NCT01470703?term=eo-lia\&rank=1. Accessed December 2014

72. SUPERNOVA trial of ECCO2-R for moderate ARDS. Available at: http://www. esicm.org/research/trials-group/supernova. Accessed December 2014

73. Paden ML, Conrad SA, Rycus PT, Thiagarajan RR. Extracorporeal Life Support Organization Registry Report 2012. ASAIO J. 2013;59:202-10.

74. Rega FR, Evrard V, Bollen $\mathrm{H}$, et al. $\mathrm{pH} 48 \mathrm{~h}$ after onset of extracorporeal membrane oxygenation is an independent predictor of survival in patients with respiratory failure. Artif Organs. 2007;31:384-9.

75. Frenckner B, Palmer $P$, Linden V. Extracorporeal respiratory support and minimally invasive ventilation in severe ARDS. Minerva Anestesiol. 2002;68:381-6.
76. Macha M, Griffith BP, Keenan R, et al. ECMO support for adult patients with acute respiratory failure. ASAIO J. 1996:42:M841-844.

77. Cordell-Smith JA, Roberts N, Peek GJ, Firmin RK. Traumatic lung injury treated by extracorporeal membrane oxygenation (ECMO). Injury. 2006;37:29-32.

78. Haneya A, Philipp A, Foltan M, et al. First experience with the new portable extracorporeal membrane oxygenation system Cardiohelp for severe respiratory failure in adults. Perfusion. 2012;27:150-5.

79. Huang YK, Liu KS, Lu MS, Wu MY, Tsai FC, Lin PJ. Extracorporeal life support in post-traumatic respiratory distress patients. Resuscitation. 2009;80:535-9.

80. Isgro S, Patroniti N, Bombino M, et al. Extracorporeal membrane oxygenation for interhospital transfer of severe acute respiratory distress syndrome patients: 5-year experience. Int J Artif Organs. 2011;34:1052-60.

81. Oshima K, Kunimoto $F$, Hinohara $\mathrm{H}$, et al. Evaluation of prognosis in patients with respiratory failure requiring venovenous extracorporeal membrane oxygenation (ECMO). Ann Thorac Cardiovasc Surg. 2010;16:156-62.

82. Bermudez CA, Rocha RV, Sappington PL, Toyoda Y, Murray HN, Boujoukos AJ. Initial experience with single cannulation for venovenous extracorporeal oxygenation in adults. Ann Thorac Surg. 2010;90:991-5.

83. Goulon M, Raphael JC, Gajdos P, Barois A, Babinet P, Margent P. Membrane oxygenators for acute respiratory insufficiency. Clinical use in 11 patients. Intensive Care Med. 1978;4:173-9.

84. Park M, Azevedo LC, Mendes PV. First-year experience of a Brazilian tertiary medical center in supporting severely ill patients using extracorporeal membrane oxygenation. Clinics (Sao Paulo). 2012;67:1157-63.

85. Park YH, Hwang S, Park HW, et al. Effect of pulmonary support using extracorporeal membrane oxygenation for adult liver transplant recipients with respiratory failure. Transplant Proc. 2012;44:757-61.

86. Rossaint R, Pappert D, Gerlach H, Lewandowski K, Keh D, Falke K. Extracorporeal membrane oxygenation for transport of hypoxaemic patients with severe ARDS. Br J Anaesth. 1997;78:241-6.

87. Brunet F, Belghith M, Mira JP, et al. Extracorporeal carbon dioxide removal and low-frequency positive-pressure ventilation. Improvement in arterial oxygenation with reduction of risk of pulmonary barotrauma in patients with adult respiratory distress syndrome. Chest. 1993;104:889-98.

88. Muellenbach RM, Kredel M, Wunder C, et al. Arteriovenous extracorporeal lung assist as integral part of a multimodal treatment concept: a retrospective analysis of 22 patients with ARDS refractory to standard care. Eur J Anaesthesiol. 2008;25:897-904.

89. Nierhaus A, Frings D, Braune $\mathrm{S}$, et al. Interventional lung assist enables lung protective mechanical ventilation in acute respiratory distress syndrome. Minerva Anestesiol. 2011;77:797-801.

90. Cho WH, Lee K, Huh JW, Lim CM, Koh Y, Hong SB. Physiologic effect and safety of the pumpless extracorporeal interventional lung assist system in patients with acute respiratory failure - a pilot study. Artif Organs. 2012;36:434-8

91. Conrad SA, Zwischenberger JB, Grier LR, Alpard SK, Bidani A. Total extracorporeal arteriovenous carbon dioxide removal in acute respiratory failure: a phase I clinical study. Intensive Care Med. 2001;27:1340-51. 